\title{
Study on Voltage Output Mechanism of Cable Sensor for Vibration Detection
}

\author{
Fumito Ito*, Dauren F. Akhmetov*, Masaaki Komazaki*, Hiroto Tachikawa**, \\ Masayuki Ujinira**, Kenji Ohara** and Youhei Kawamura***
}

\begin{abstract}
The characteristics and applications of the "cable sensor," a new type of vibration sensor, are considered in this paper. Previous studies by the authors on the practical use of cable sensors in rockfall detection are reviewed. The mechanism of output voltage generation by the sensor is discussed. Methods for analyzing static electricity and molecular dynamics are applied toward elucidating the sensor's measurement principles. The following results were obtained:

1) Alternating output voltage of cable sensor occurs when an external vibration source causes multiple contacts between the conductor part of the sensor and its dielectric material (FEP). 2) The above fact is demonstrated experimentally through a vibration test using an audio speaker and an acceleration sensor. Output waveforms of the cable sensor are similar to those of the acceleration sensor. 3) From molecular dynamics analysis, it was supposed that $\mathrm{FEP}$ with a higher electron affinity might be produced by adding a $-\mathrm{CF}_{2} \mathrm{CF}_{3}$ group to the main chain of FEP in order to increase the output voltage of the sensor. 4) Output voltage of the sensor decreases with increase in capacitance of the circuit. This means that the output voltage decreases both with an increase in the length of the cable sensor and with the introduction of an extension signal transmission cable. The signal decrement could be compensated by amplification. A transfer function of the measurement circuit is derived, which enables evaluation of the necessary amplification gain. The results of the analysis of material combination for the sensor components and the proposed solutions to the equation describing the measurement circuit have deepened the understanding of the voltage output mechanism and measurement principles of cable sensors.
\end{abstract}

Key Words : cable sensor, vibration detection, mechanism of output generation, output chacracteristies

\section{Introduction}

Many unstable slopes are found along coastal and mountainous routes, particularly in northern Japan, and in Italy, Austria and Switzerland (P. Paronuzzi, 1989¹) A. Azzoni, 19952)). In 1996, a slope failure of $11,000 \mathrm{~m}^{3}$ occurred on National Route 229 in Hokkaido Prefecture, Japan (Y. Ishijima et al., 19973) ). Nineteen bus passengers died in this large-scale rock failure. Development of systems to predict rockfall or slope failure is strongly required for prevention of such tragic accidents. It has been shown (e.g., M. Hirano et al., 19894), S. Kamio et al., $1999^{5)}$ ) that small rockfalls often precede large-scale slope failures.

In light of the above, the authors have developed a rockfall monitoring system composed of string cable sen-

* NTT DoCoMo Hokkaido Inc., Sapporo

** Graduate School of Engineering, Hokkaido University, Sapporo

*** Institute of Engineering Mechanics and Systems, University of Tsukuba, Tsukuba

(Received June 14, 2004)

(Revised June 4, 2005) sors for vibration detection and a mobile multimedia network for signal transmission. Preliminary sensitivity tests of the sensor were carried out (M. Ujihira et al., $\left.1998^{6}\right)$ ) when 14 strings of cable sensor were installed on the internal surface of a model rock shed. The sensor registered an output signal of about $1.0 \mathrm{~V}$ when a steel ball of $7.26 \mathrm{~kg}$ was dropped from the height of $1.5 \mathrm{~m}$. It was confirmed that cable sensors could be used to reproduce the trajectory of the rockfall and could give information on the impact source when the sensor was laid in a mesh pattern (M. Komazaki et al., 19997)). The tensile strength $(400 \sim 500 \mathrm{~N})$ of the original cable sensor was insufficient for field use. To overcome this drawback, the authors developed a steel wire cable sensor, in which the hemp chord at the center of the steel wire was replaced by a cable sensor. This raised the tensile strength about 150 times to $75 \mathrm{kN}$ (M. Ujihira et al., 19998)). Application tests were carried out with remodeled steel wire cable sensors stretched over a slope. It was confirmed that the rockfall frequency, origin of rockfall trajectory and rockfall energy could be evaluated from the measured waveforms (M. Ujihira et al., 20019)).

However, a detailed explanation of the cable sensor 
measurement principles still remains a topical issue for popularization of the proposed rockfall detection system. This paper uses methods for analyzing static electricity and molecular dynamics to study the voltage output mechanism and characteristics of cable sensors. A solution for calculating electron flow is derived, which allows proposals for sensitivity improvements to cable sensors.

\section{Structure of Cable Sensor}

Fig. 1 (a) is a schematic of the cable sensor. This sensor is composed of four layers in a coaxial configuration that is as same as that of the coaxial cables typically used for signal transmission. The $1^{\text {st }}$ layer, in the center of Fig. 1( a ), is a twisted conductor. The $2^{\text {nd }}$ layer is a dielectric substance made of polyfluoroethylene (FEP), which is a co-polymer composed of tetrafluoroethylene and fluoropropylene. The $3^{\text {rd }}$ layer is the shield net, and the $4^{\text {th }}$ is the insulation material. There is a little clearance between the $1^{\text {st }}$ and $2^{\text {nd }}$ layers. The cable sensor vibrates when an impact or elastic wave is given from outside. It is considered that electrons flow instantly between the central conductor and the dielectric substance when the conductor contacts the internal surface of the FEP. A difference of electric potential occurs between the conductor and the shield net because the $2^{\text {nd }}$ layer works as a capacitor. Output voltage between the ends can be detected when a resistance is introduced between the central conductor and the shield net of the $3^{\text {rd }}$ layer. As mentioned above, the tensile strength of the cable sensor (CS) is about $400-500 \mathrm{~N}$, which is insufficient for usage in the field. For this reason, the authors developed the steel wire cable sensor (SWCS) shown in Fig. 1(b), in which the original cable sensor is coaxially rolled in steel wire

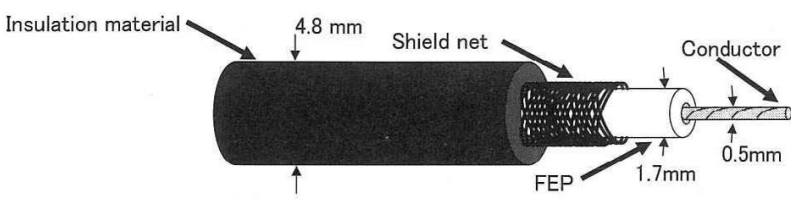

(a) Cable sensor (CS)

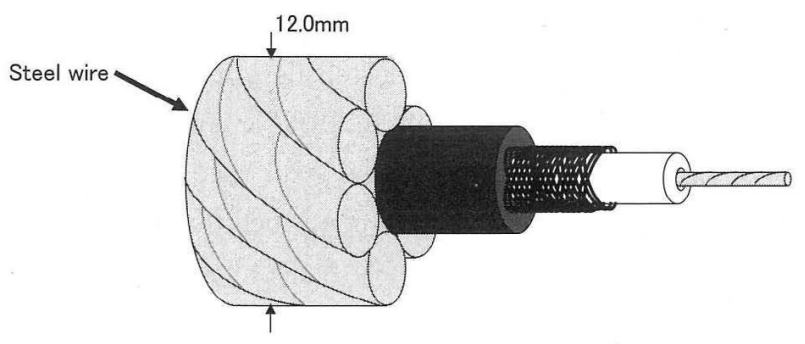

(b) Steel wire cable sensor (SWCS)

Fig. 1 Schematic view of cable sensor structure using a twining machine. The diameter after rolling is 12 $\mathrm{mm}$. The voltage output characteristics of both sensors will be described in the following chapter.

\section{Mechanism of Voltage Output}

\section{1 Physical description of cable sensor operation}

According to well-known research on static electricity ${ }^{10), 11}$, the principle of output voltage generation by cable sensor can be described as follows. The conductor shown in Fig. 1 consists of 7 twisted strings with an overall diameter of about $0.5 \mathrm{~mm}$. When an external impact is applied to the sensor, the central conductor starts to vibrate inside the FEP tube. Fig. 2 is a schematic of electric charge generation when a metal makes contact with the polymer surface. The symbols are explained under the figure. Fig. 2(a) shows the state before contact: The vertical axis is the energy level of electron, and the horizontal axis is the depth from the contact surface. The suffix M means metal conductor and I means FEP insulation material. The energy level in a vacuum is expressed as $E=0$. The energy level of conductive band and the Fermi level are expressed by $E_{C}$ and $E_{F}$, respectively. Fig. 2(b) shows the state of energy level of electron just after the contact of two kinds of material. It can be understood that in this case, the Fermi level of the metal becomes equal to that of the dielectric material $^{10)-14)}$.

In this case, the work function of the copper used for a conductor with $\phi_{M}=4.54 \mathrm{eV}^{10)}$ and the work function of FEP with $\phi_{I}=5.80 \sim 6.03 \mathrm{eV}^{12}$. This means that the Fermi level of the conductor is higher than that of FEP. For this reason, electrons flow from conductor to FEP when two kinds of material contact each other. Removed electrons are mainly captured on the surface of FEP. For further movement of the electrons, the energy level of the
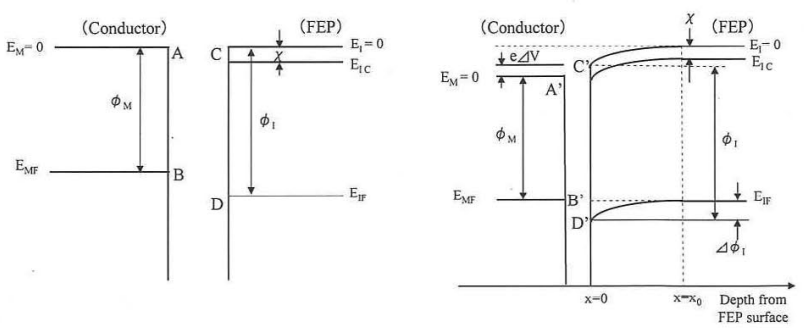

$E_{\mathrm{y}}$ : Energy level of meta

$\mathrm{E}_{\mathrm{M}}:$ Fermi level of metal

$\mathrm{E}_{1}$ : Energy level of FEP

$\mathrm{E}_{\mathrm{IC}}$ : Low boundary of conductivity zon

$E_{1 E}:$ Fermi level of FEP

(a) Before contact

$\phi$ W Work function of metal

$\phi_{1}$ : Work finction of FEP

$x:$ Energy of electron affinity $\Delta \phi_{1}$ : Gap between work functions $\phi_{\mathrm{M}}$ and $\phi_{1}$

$x_{0}$ : Depth of FEP layer influenced by metal

(b) After contact

Fig. 2 Principle of electric charge generation in cable sensor 
superficial layer must be lower than that of the deep FEP layers (Fig. 2(b)). The layer in which a curving of the energy band occurs is called the "thin layer of electric charge" (Fig. 3(a)). The depth of the layer is considered to be on the order of $10^{-9} \mathrm{~m}^{10)}$. The metal surface takes a positive elective charge $(+)$ and the FEP surface takes a negative electric charge (-) when $\phi_{M}<\phi_{I}$ is satisfied. Electric charge per unit area $\sigma_{s}\left[\mathrm{C} / \mathrm{m}^{2}\right]$ can be given as follows (H. Bauser et al., 197013), Y. Wada 1971 ${ }^{14)}$ ) :

$$
\sigma_{S}=e \cdot D_{s} \cdot \Delta \phi_{I}
$$

Here, $D_{s}\left[\mathrm{eV}^{-1} \cdot \mathrm{m}^{-2}\right]$ is the density of Fermi energy level per unit area, $e[\mathrm{C}]$ is the electric charge per electron. As mentioned above, the Fermi level on the surface side of FEP must drop from $B^{\prime}$ to $D^{\prime}$ (Fig. 2(b)) in order for the FEP surface to accept electrons moving from metal when the Fermi level of metal $E_{M F}$ drops to $E_{I F}$. The following expression for work function between conductor and FEP can be given using the gap $\Delta \phi_{I}$ (Y. Wada, 1971 $\left.{ }^{14)}\right)$ :

$$
\phi_{M}+e \cdot \Delta V=\phi_{I}-\Delta \phi_{I}
$$

It is considered that contact surfaces of metal and FEP form a condenser with a capacitance per unit area of $C_{A}$ $\left[\mathrm{F} / \mathrm{m}^{2}\right]$, and a potential gap of $\Delta V$ is observed between two kinds of surfaces. The $C_{A}$ can be written as ${ }^{14)}$

$$
C_{A}=\varepsilon_{0} / x
$$

Equation ( 3 ) can be re-written as Equation (4), where $\varepsilon_{0}$ is the dielectric constant and $x$ is a small gap between contact surfaces, which are considered uneven and rough at a scale on the order of $10^{-9} \mathrm{~m}$.

$$
\Delta V=\sigma_{S} \cdot x / \varepsilon_{0}
$$

The following equation can be deduced from Equations (1), ( 2 ) and ( 4 ).

$$
\sigma_{S}=e \cdot D_{S}\left(\phi_{I}-\phi_{M}\right) /\left(1+e^{2} D_{s} x / \varepsilon_{0}\right),
$$

where the numerical values of each variable in the right hand term of Equation ( 5 ) are as follows ${ }^{13), 14)}$

$$
\begin{aligned}
& e=1.602 \times 10^{-19}[\mathrm{C}], D_{s}=1 \times 10^{16}\left[\mathrm{eV}^{-1} \cdot \mathrm{m}^{-2}\right], \\
& \varepsilon_{0}=8.85 \times 10^{-12}[\mathrm{~F} / \mathrm{m}], x=1 \times 10^{-9}[\mathrm{~m}]
\end{aligned}
$$

Equation ( 5 ) can be more briefly expressed as Equation

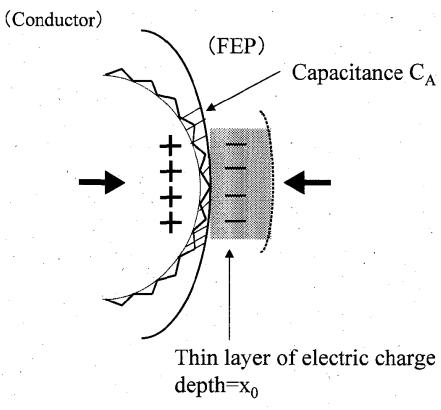

(a) Contact process

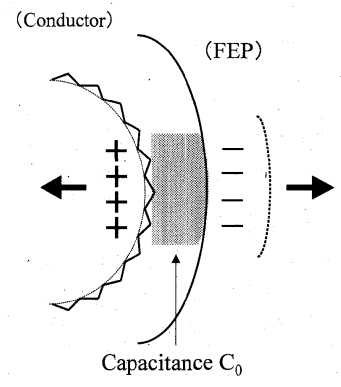

(b) Separation process
Fig. 3 Schematic view of electric charge generation process
( 6 ), because $e^{2} D_{s} x / \varepsilon_{0}$ in the right hand term of Equation (5) is nearly equal to 0 when the above values are substituted in

$$
\sigma_{S}=e \cdot D_{S}\left(\phi_{I}-\phi_{M}\right)
$$

Equation (6) means that electric charge per unit area $\sigma_{s}$ has a linear relationship with both the density of Fermi energy level per unit area and with the gap of the work function $\left(\phi_{I}-\phi_{M}\right)$.

Electrons will flow in reverse from FEP to metal when metal and FEP separate from each other. It is considered that capacitance $C_{0}$ appears between separated surfaces (Fig. 3(b)), and an electric charge of $q_{0}$ is stored between the surfaces initially, which is expressed as

$$
q_{0}=S \sigma_{S}
$$

where $S$ is the contact area.

This paper points out that the flow of electric charge is the origin of output voltage from cable sensor. Therefore, the combination of materials with significant difference of Fermi levels is the most important matter in achieving satisfactory output from cable sensor.

\section{2 Measurement circuit and voltage output charac- teristics}

In this section, the authors confirm experimentally that the voltage output of a cable sensor results from the mechanical contact of two materials with different Fermi levels. Then the influence of the transmission circuit on the output signal of the cable sensor is discussed.

Fig. 4 shows the equivalent scheme for measurement circuit using a cable sensor. The origin of electron flow, which is an electric charge generator, is indicated by the mark $\theta . C_{0}$ is the capacitance between contact surfaces of small area, $C_{1}$ is the capacitance between contact point and the outside of the FEP tube, and $C_{c}$ is the capacitance between metal conductor and shield net outside the FEP, whose value is proportional to the cable sensor length. $R$ is the load resistance between conductor and shield net. Output voltage is measured at the resistance $R$.

Applying Kirchhoff's law to the circuit, the following equation is obtained.

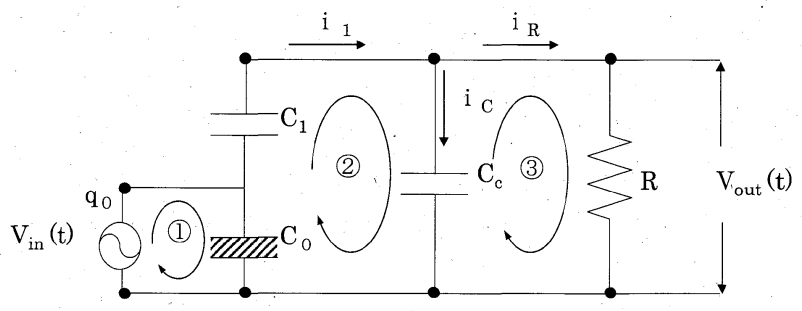

Fig. 4 Equivalent scheme of measurement circuit using cable sensor 


$$
\frac{d v_{\text {out }}(t)}{d t}+\frac{v_{\text {out }}(t)}{R\left(C_{c}+C_{1}\right)}=\frac{C_{1}}{\left(C_{c}+C_{1}\right)} \frac{d v_{\text {in }}(t)}{d t}
$$

The characteristics of cable sensor were first studied experimentally. Fig. $\mathbf{5}$ shows a view of the vibration test of the original cable sensor, the redesigned steel wire cable sensor, and the accelerometer, where a pulse generator and an audio speaker produce the mechanical vibration.

Fig. 6 shows three kinds of waveform obtained from the vibration tests illustrated in Fig. 5, where mechanical vibration of $500 \mathrm{~Hz}$ was given. Fig. 6(a) shows the output waveform of the CS under the experimental conditions shown in Table $1: C_{1}=6.3 \mathrm{pF}$ and $C_{C}=693.6 \mathrm{pF}$. It was supposed that only a $10 \mathrm{~cm}$ piece of the cable sensor's $11 \mathrm{~m}$ contributed to electric charge generation. Fig.6(b) shows the output waveform of SWCS measured under the same circuit conditions. Both waveforms have the cyclic forms of $500 \mathrm{~Hz}$. The amplitudes in Fig. 6( a ) and 6(b) are roughly the same, although the output fluctuation is slightly greater for the SWCS. This means that the stren-

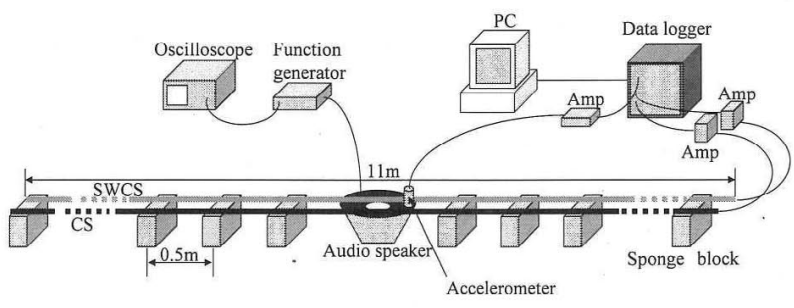

Fig. 5 Schematic view of laboratory vibration test

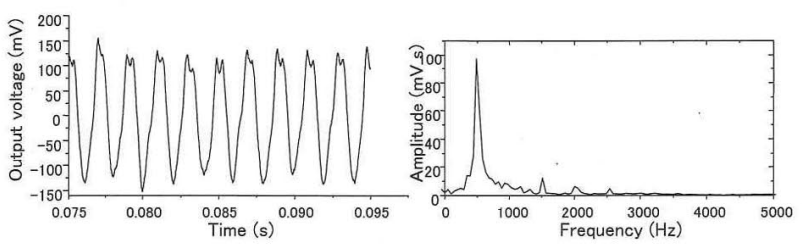

(a) Original cable sensor (CS)
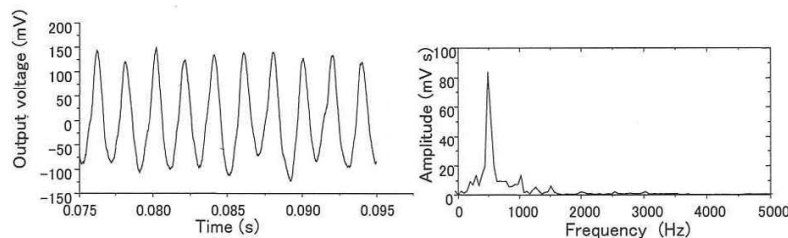

(b) Steel wire cable sensor (SWCS)
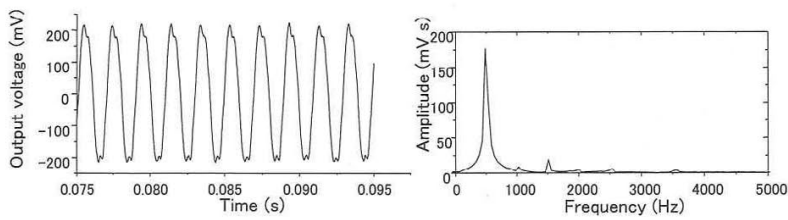

(c) Accelerometer

Fig. 6 Waveforms and frequency spectra of sensor' output voltage (mechanical vibration of $500 \mathrm{~Hz}$ was given) gthened SWCS can be used instead of the CS. Fig. 6( c ) shows the accelerometer output waveform registered simultaneously with the signals from the cable sensors. The acceleration signal is considered as a standard one, for comparison with the output of cable sensors. As seen from Fig. $6(\mathrm{a} \sim \mathrm{c})$, when a mechanical vibration of $500 \mathrm{~Hz}$ is applied to sensors, the three output waveforms look alike, although a phase gap is observed. Fourier spectra of three kinds of the waveforms have almost the same frequency components in the similar ratio. This alternative waveform proves that alternative flow of electric charge is the origin of $v_{\text {in }}(t)$ in Equation ( 8$)$, due to alternating contact and separation of the conductor and the internal surface of FEP tube.

Output voltage $v_{\text {out }}=b e^{j(\omega t+\phi)}$ can be obtained theoretically when the input of $v_{\mathrm{in}}=a e^{j \omega t}$ is given to the circuit. Then the transfer function $G(j \omega)$ can be expressed as

$$
|G(j \omega)|=\frac{C_{1} R}{\sqrt{1 / \omega^{2}+R^{2}\left(C_{c}+C_{1}\right)^{2}}}
$$

According to the right-hand term of Equation ( 9 ), the output voltage depends on signal frequency and capacitance values $C_{1}$ and $C_{c}$, where $C_{1}$ is small and constant. Fig. 7 shows the relationship between the signal frequency and

Table 1 Transfer function calculation conditions without any transmission cable

\begin{tabular}{c|c|c}
\hline \hline Parameter & Value & Comment \\
\hline$C_{1}$ & $6.36 \mathrm{pF}$ & $L=10 \mathrm{~cm}$ \\
\hline$C_{c}$ & $693.64 \mathrm{pF}$ & $L=10.9 \mathrm{~m}$ \\
\hline$R$ & $1 \mathrm{M} \Omega$ & \\
\hline
\end{tabular}

Table 2 Characteristics of extension transmission cables

\begin{tabular}{c|c|c}
\hline \hline Extension cable & $\begin{array}{c}\text { Dielectric capacitance } \\
(\mathrm{nF} / 100 \mathrm{~m})\end{array}$ & $\begin{array}{c}\text { Resistance } \\
(\Omega / 100 \mathrm{~m})\end{array}$ \\
\hline Coaxial cable 1 & 5.0 & 7.5 \\
\hline Coaxial cable 2 & 5.8 & 7.5 \\
\hline Coaxial cable 3 & 7.5 & 7.5 \\
\hline Twisted pair cable & 36.5 & 2.8 \\
\hline
\end{tabular}

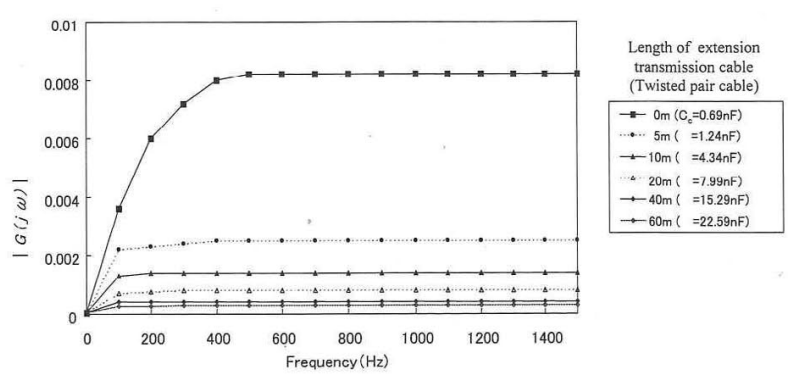

Fig. 7 The relationship between transfer function $|G(j \omega)|$ and frequency 
the calculated value of $|G(j \omega)|$ when the capacitance of extended transmission cable (twisted pair cable in Table 2) of 5-60 $\mathrm{m}$ was added to original capacitance $C_{c}$. It is seen from the figure that the output voltage decreases with increase in capacitance $C_{c}$. It is evident that use of the transfer function (Equation (9)) allows calculation of the amplification rate necessary to achieve satisfactory output level.

A laboratory test was carried out to estimate the influence of extension transmission cable connected in series with an $11 \mathrm{~m}$-length cable sensor. Here, also a coaxial cable with a capacitance of $5.8 \mathrm{nF} / 100 \mathrm{~m}$ was used

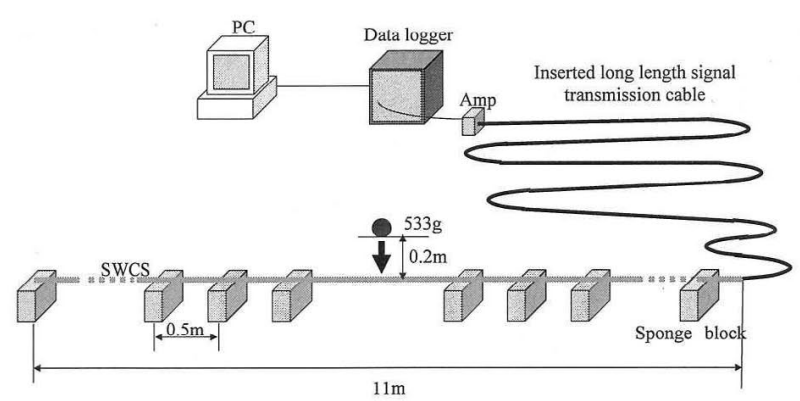

Fig. 8 Experiment sctup for estimation of transmission cable influence

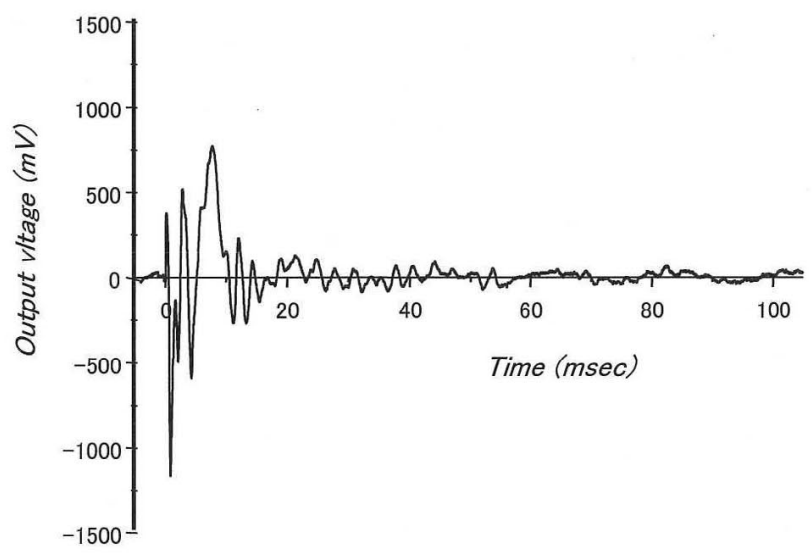

(a) Wave form

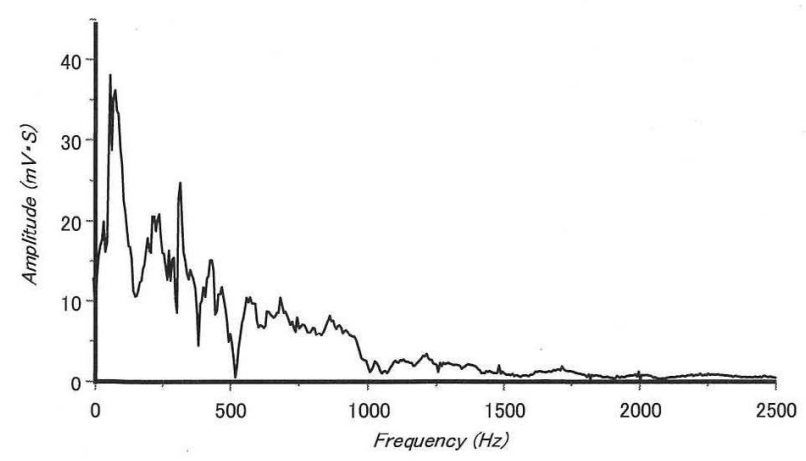

(b) Fourier spectrum

Fig.9 An example of measured waveform and its Fourier spectrum (coaxial cable 2, Table 2). The experimental setup is shown in Fig. 8, where the SWCS is used, which features almost the same output characteristics as the conventional cable sensor and still is suitable for practical application. A $533 \mathrm{~g}$ steel ball was dropped directly onto the steel wire cable sensor from a height of $20 \mathrm{~cm}$. Experimental conditions are close to that encountered in the field. Fig. 9 shows an example of measured waveform of the steel wire cable sensor and its Fourier spectrum. It is seen that the output voltage exceeds $1000 \mathrm{mV}$ and the dominant frequency is around $100 \mathrm{~Hz}$.

Fig. 10 shows the relationship between the relative sig. nal decrement $v_{0_{-} x m} / v_{0_{-} 0 m}$ and the length of signal transmission cables, where $v_{0}$ om is the output voltage without extended transmission cable and $v_{0_{-} x m}$ is the output voltage when a transmission cable (coaxial cable 2) of $x$

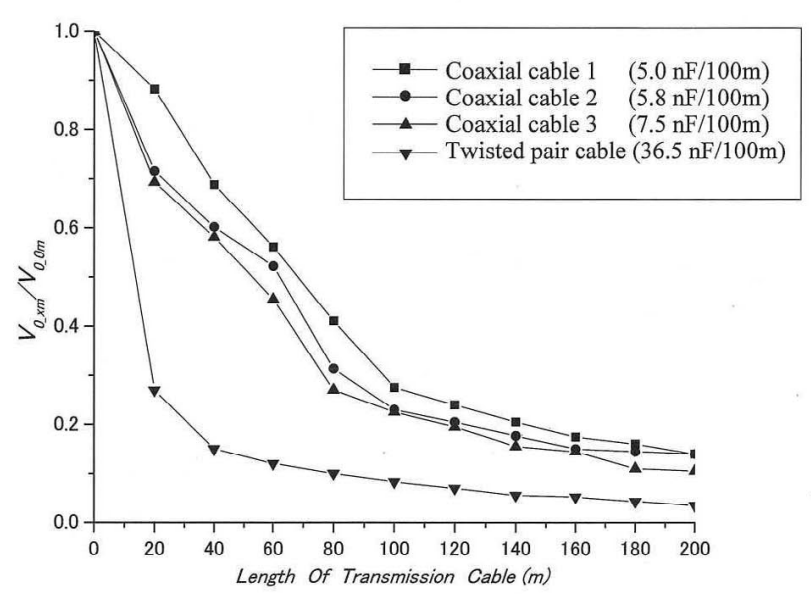

Fig. 10 Relationship between the signal decrement and the length of signal transmission cable

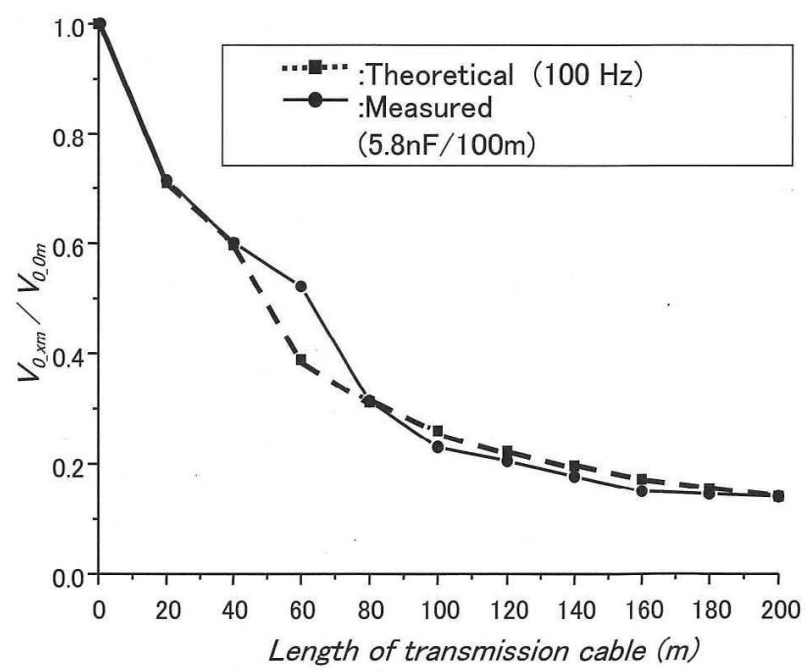

Fig. 11 Measured and theoretical relationships between relative signal decrement and the length of transmission cable (coaxial cable 2) 
meters in length is used. Fig. 11 shows two relationships between relative signal decrement $v_{0 \_} x m / v_{0} 0 m$ and the length of signal transmission cable. The solid line is an experimental curve, and the dotted line is a theoretical result calculated from Equation ( 9 ) for fixed frequency value, corresponding to the dominant frequency of $100 \mathrm{~Hz}$. It is assumed that the length of cable is expressed by the value of the circuit capacitance $C_{c}$.

According to Fig. 11, the value of $v_{0 \_} x m / v_{0 \_} 0 m$ decreases with increase of the length of signal transmission cable. The decreasing trend is quite similar to that of $|G(j \omega)|$ calculated against the input of $v_{\text {in }}=a e^{j \omega t}$. Due to this output characteristic, it is necessary to amplify the output, in proportion to the length of SWCS or signal transmission cable when the signal transmission cable is inserted before the resistance $R$.

In this section, the equivalent circuit for cable sensor is proposed and two examinations are discussed.

The vibration test using an audio speaker proved that alternating flow of electrons was caused by the alternating contact and separation of conductor with the internal surface of FEP tube.

From calculation of the value of the transfer function and simulation-based analysis, it was confirmed that the output voltage decreases with increase in capacitance $C_{C}$, i.e. with increase in the length of cable sensor. For practical applications, an amplifier should be used to get an output level that matches the capacitance $C_{c}$. The amplification gain can be evaluated using the value of $|G(j \omega)|$.

It was confirmed from the experiment in which a steel ball was dropped onto the SWCS that the voltage output decreases with the increase in capacitance $C_{C}$ in the manner shown by the calculated curves, although the measured waveform contained various frequency components.

\section{Theoretical Study on Electronic States of Per- Fluorocarbon Polymer (Neoflon)}

In the preceding chapter, contact flow of electric charge between metal and FEP surfaces was explained using the concept of work function and Fermi level. In this chapter, the authors carry out further investigation related to the mechanism of electron interaction of FEP with metal surface using the concepts of electron affinity, ionization potential and band gap. We use semi-empirical molecular orbital (MO) theory at the PM3 level ${ }^{15)}$ to calculate the structure and electronic states of FEP. The FEP used in the present study is a per-fluorocarbon polymer (called
Neoflon), which is a co-polymer of tetrafluoroethylene and fluoropropylene. The chemical structures of these molecules are illustrated in Fig. 12. Four-monomer-unit FEP $(n=4)$ was used as a model compound of FEP. For comparison, the electronic states of polypropylene were investigated in the same manner.

\section{1 Molecular structure of FEP}

First, the molecular structure of the model compound (4-monomer-unit FEP) was fully optimized accordingly to the theory at the PM3 level. The optimized structure is illustrated in Fig. 13(a). The structure is slightly bent from the linear structure because of the repulsive interac tion between trifluoromethyl groups $\left(\mathrm{CF}_{3}{ }^{-}\right)$in the side chains.

\section{2 Electron affinity and ionization potential}

Using the optimized structure, electron affinity (EA) and ionization potential (IP) of the model compound were calculated for the anionic and cationic states. EA and IP are defined by Equations (10) and (11).

$$
\begin{aligned}
& \mathrm{FEP}+\mathrm{e}^{-} \rightarrow(\mathrm{FEP})^{-}+\mathrm{EA} \\
& \mathrm{FEP}+\mathrm{IP} \rightarrow(\mathrm{FEP})^{+}+\mathrm{e}^{-}
\end{aligned}
$$

EA refers to the formation energy of FEP anion from neutral FEP by electron attachment, and IP refers to the energy required for the formation of cationic state of FEP. If EA has a positive value, the reaction in Equation (10) is exothermic. The calculated EA and IP for FEP are shown in Table 3, together with those for polypropylene. FEP has
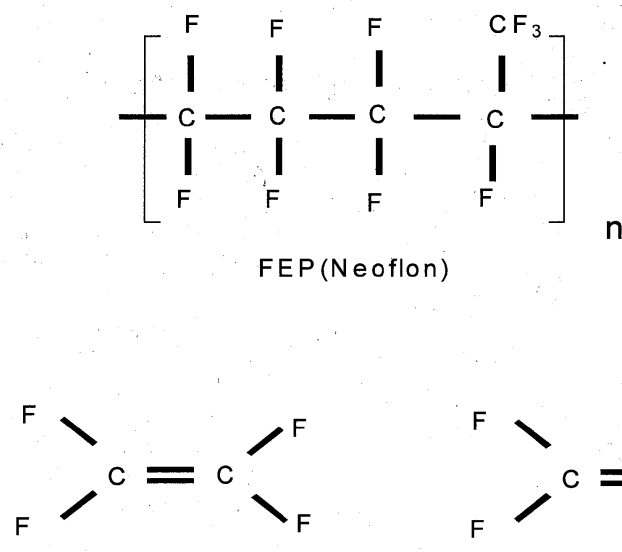

tetrafluoroethylene
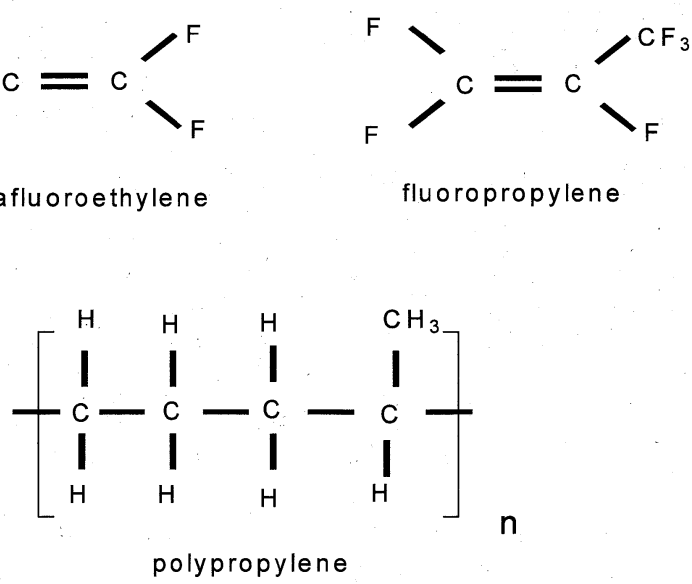

Fig. 12 Chemical structures of conventional materials 


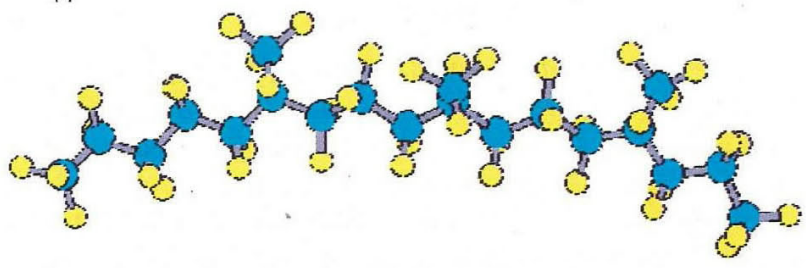

(a) Optimized structure

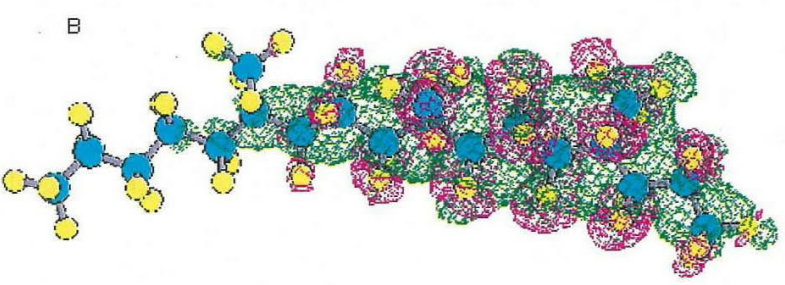

(b) Orbit of excessive electron

Fig. 13 Optimized structures of FEP

Table 3 Electron affinities (EA), ionization potentials (IP) and band gaps of neoflon and polypropylene calculated with the semi-empirical PM3 method

\begin{tabular}{c|c|c}
\hline \hline & FEP & polypropylene \\
\hline Electron affinity, EA [eV] & +2.63 & -2.79 \\
\hline Ionization potential, IP [eV] & 13.53 & 10.85 \\
\hline Band gap, Eg [eV] & 6.69 & 9.15 \\
\hline
\end{tabular}

a positive electron affinity $(+2.63 \mathrm{eV})$, and its ionization potential $(13.53 \mathrm{eV})$ is higher than that for polypropylene. The higher electron affinity of FEP originates from the abundance of $F$ atoms in side and main chains. These characteristics greatly differ from those of polypropylene, in which the electron affinity is negative $(-2.79 \mathrm{eV})$.

The band gap of FEP was calculated as $6.69 \mathrm{eV}$ at the PM3-Cl level. The band gaps ( $\mathrm{Eg}$ ) are estimated from the first excitation energies between $S_{0} \rightarrow S_{1}$ states by PM3-CI method. This magnitude indicates that FEP acts as an insulator. The orbit of excess electrons in FEP anion radicals is illustrated in Fig. 13 ( b ). It is seen that excess electrons are mainly distributed around the $\mathrm{CF}_{3}-$ side chain, and this is why the branched polymer composed of $\mathrm{CF}_{3}{ }^{-}$groups is more effective at generating an output voltage.

\section{3 Electron transfer between FEP and metal surface}

The present study indicates that electrons are easily transferred from metal to FEP when FEP contacts a metal surface. This is due to the high electron affinity of FEP (Neoflon). The excess electrons in the FEP anion radical are mainly distributed on $\mathrm{CF}_{3}$-branched side chains. This result suggests that the polymer, composed of many $\mathrm{CF}_{3}$ groups in side chains, possess high performance as an FEP

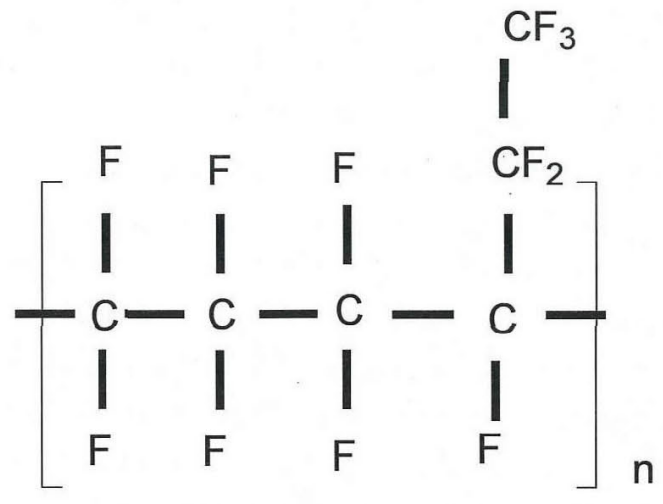

(a) modified FEP ( I )

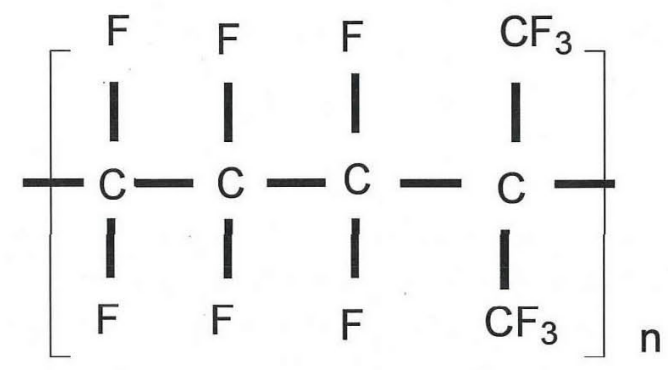

(b) modified FEP (II)

Fig. 14 Molecular design of new material for FEP

Table 4 Electron affinities (EA), ionization potentials (IP) of modified FEP calculated with the semi-empirical PM3 method

\begin{tabular}{c|c|c}
\hline \hline & Type I & Type II \\
\hline Electron affinity, EA [eV] & +2.90 & +2.61 \\
\hline Ionization potential, IP [eV] & 13.66 & 13.50 \\
\hline
\end{tabular}

polymer.

\section{4 Molecular design of new material for FEP}

As mentioned above, a polymer used as FEP must have a positive electron affinity. The present calculations show that EA of FEP is $+2.63 \mathrm{eV}$. In this section, on the basis of $\mathrm{MO}$ calculation, we propose a new polymer with a higher EA than FEP.

Two types of polymers were examined for trial compounds (Fig. 14). The EA and IP calculated for the modified FEP (I) and (II) are given in Table 4. The modified FEP (I) has a positive electron affinity $(+2.90$ $\mathrm{eV}$ ), and the value is much larger than that of original FEP $(+2.63 \mathrm{eV})$. The EA of modified FEP (II) is +2.61 $\mathrm{eV}$, which is comparable to that of the original FEP.

In the study described above, the molecular structures and electronic states of FEP were determined by semiempirical PM3 MO calculations. The model compound composed of four-monomer-unit FEP was applied. The calculations showed that FEP had a positive electron 
affinity that is large enough to accept excess electrons. When FEP contacts the metal surface, electrons are transferred from metal to FEP. The excess electrons are mainly distributed to the trifluorocarbon group $\left(\mathrm{CF}_{3}{ }^{-}\right)$of FEP. The PM3-MO calculations predicted that modification of FEP (by addition of the $-\mathrm{CF}_{2} \mathrm{CF}_{3}$ group to the main chain) could achieve a high-performance FEP.

It is shown in this section that molecular dynamics analysis provides a detailed explanation of the nature of output voltage of cable sensor. Practical application of the results requires intensive experimental work to design an improved cable sensor and to confirm its superiority to the conventional one. This is an object of further research.

\section{Conclusion}

This paper reviewed preceding studies of the authors on the practical use of cable sensors for rockfall detection and described a possible approach to explanation of the precise mechanism of output voltage generation for cable sensors. The results of this study are as follows:

1) A hypothesis was proposed, from theoretical studies using the concepts of work function and electron affinity. The hypothesis is that alternating output voltage is generated by contact and separation between a conductor part and the dielectric material (FEP) of a cable sensor.

2) The hypothesis was tested and proved by a vibration test using an audio speaker and an acceleration sensor. Output waveforms measured by steel wire cable sensor were similar to those measured by acceleration sensor.

3) According to the theory-based calculation and experimental test of output characteristics of equivalent circuit for cable sensor, the output voltage decreases with increase in the capacitance of the circuit. This means that output voltage decreases when a long cable sensor is used or when a signal transmission cable is connected before the output resistance $R$. This drawback could be compensated by amplifier use. The amplification gain could be evaluated through analysis of the transfer function of the equivalent circuit.

4) From molecular dynamics analysis, it was proposed that FEP of higher electron affinity could be produced by adding a $-\mathrm{CF}_{2} \mathrm{CF}_{3}$ group to the main chain of FEP. Experimental verification of this method is an object of further research.

\section{References}

1) P. Paronuzzi : Probabilistic approach for design optimiza tion of rockfall protective barriers, Quarterly Journal of Engineering Geology, Londọn, 22, 175/183 (1989)

2) A. Azzoni, G. LaBarbera and A. Zaninetti : Analysis and prediction of rockfalls using mathenhatical model, Int. J. of Rock Mechanics \& Mining Science, 32-7, 709/724 (1995)

3) Y. Ishijima and Y. Fujii : Study of the mechanism of slope failure at Toyohama tunnel, 10 February, 1996, Int. J. Rock Mech. Min. Sci., 34(3/4), p. 519, Paper No. 87 (CD-ROM) (1997)

4) M. Hirano, H. Suwa, T. Fujita, K. Okunishi and T. Ishii : Investigation of cliff collapse process at the Echizen coast rock fall hazard in 1989, Annuals, Dizas. Prev. Inst., Kyoto Univ., 33, B-1, 1/18 (1989)

5) S. Kamio, H. Yoshimatsu, R. Tsunai and Y. Wakisaka: Rock failure at No. 2 Shiraito tunnel along national route 229, Civil Engineering Journal, Public Works Research Inst., Ministry Construction, 39-11, 2/5 (1997)

6) M. Ujihira, S. Suzuki, S. Hosoya, H. Saeki, M. Kawakita and S. Sarata: Source location of impact source of weight dropping on a model tunnel using cable sensor, Journal of Japan Soc. Eng. Geol., 39-5, 423/432 (1998)

7) M. Komazaki, K. Hirama, M. Ujihira, S. Suzuki, H. Saeki and Y. Kawamura: A field test of a rock fall detection system using cable sensor and mobile multimedia network, Int. J. of Surface Mining, Reclamation and Environment, 13-4, 159/163 (1999)

8) M. Ujihira, Y. Kawamura, M. Sato, H. Konno, M. Komazaki and K. Hirama: Study on output characteristics and mechanical strength of the cable sensor for vibration detection, Proceedings of the 8th Int. Symposium on Mine Planning and Equipment Selection, 539/544 (1999)

9) M. Ujihira, F. Ito, T. Tokunaga, Y. Kawamura, S. Konno, K. Higuchi and M. Komazaki : Evaluation of the weight of fallen object using the waveforms measured by cable sensor and mobile multimedia network system, Proceedings of the 11th Int. Symposium on Mine Planning and Equipment Selection, 57/62 (2001)

10) Seidenkigakkai : Seidenki Handbook, Ohm-shya, Tokyo, 55/86 (1981)

11) Koubunshi gakkai: Handbook of Static Electrification, Chijin shokan, Tokyo, 38/71 (1982)

12) N. R. Rajopadhye and S. V. Bhoraskar: Ionization potential and work function measurements of PP, PET and FEP using low-energy electron beam, J. Material Science Letters, 5-6, 603/605 (1986)

13) H. Bauser, W. Klopffer and H. Rabenhorst: On the charging mechanism of insulating solids, Advances in Static Electricity, 1, Brussels Auxillia, 2/9 (1970)

14) Y. Wada: Kobunshi-no-kotaibussei, Baifuukan, $340 / 343$ (1971)

15) M. J. Frisch et al. : Ab-initio and semi-empirical MO Program, Gaussian 98, Revision A. 11.2, Gaussian Inc., Pittsburgh PA. (2001) 


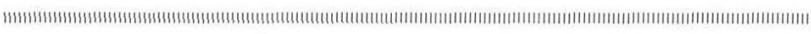
Fumito Iто (Member)

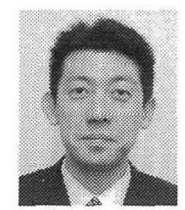

He received the B.E. degrees in engineering, department of electrical and information engineering from Tohoku University, Miyagi, Japan in 1993. Since October 2000, he has been with NTT DoCoMo, Inc., where he is a system engineer. His research interests are rock fall detection system and remote monitoring system.

\section{Dauren F. Акнметоу}

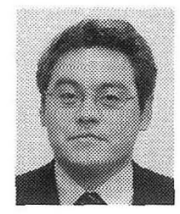

He received a Dipl. in engineering and a Candidate of Sciences (Ph.D.) degree from Kazakh National Technical University, Almaty, Kazakhstan, and a Dr. Eng. degree from Muroran Institute of Tech., Muroran, Japan in 1981, 1989 and 1999 respectively. Since April 1994 he has been with Intelligent Control Laboratory, Dept. of Computer Science and Systems Engineering, Muroran Institute of Tech.. From 2000 he is with Mobile Multimedia Development Office, NTT DoCoMo Hokkaido, Inc. His current research interests include intelligent information processing, system identification, and remote control with applications over mobile communication networks.

\section{Masaaki Konazaki}

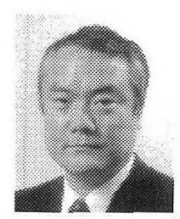

He received the B.E. degree in civil engineering and the Ph.D. degree in mineral resources development engineering from Hokkaido University, Sapporo, Japan in 1971 and 2001 respectively. Since 1991 he has been with NTT East. From 1996 he has been with NTT DoCoMo Hokkaido, Inc. Group. His research interests are remote sensing and monitoring with applications of mobile communication.
Hiroto Tachikawa

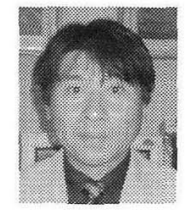

He received the B.E., M.E. and Ph.D. degrees in quantum chemistry from Hokkaido University, Sapporo, Japan in 1985, 1987 and 1994 respectively. Since 1990, he has been research associate in Division of Material Chemistry, Graduate School of Engineering, Hokkaido University. His field of work concerns molecular design of high-performance materials based on quantum theory and molecular dynamics technique.

\section{Masuyuki UjIHIRA (Member)}

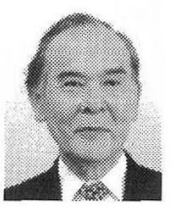

He received the B.E., M.E. and Ph.D. degrees in mineral resources development engineering from Hokkaido University, Sapporo, Japan in 1968, 1970 and 1983 respectively. Since 1975, he has been with Hokkaido University, where he is currently an associate professor. His research interests are geo-measurement engineering and diagnosis of heavy machinery for mining and construction works.

Kenji OHARA

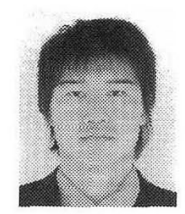

He received the B.E., M.E. degrees in mineral resources development engineering from Hokkaido University, Sapporo, Japan in 2003 and 2005 respectively. Since April 2005, he has been with Nissan motor Co., LTD., where he is a research engineer. His research interests are development of fuel cell and geo-measurement engineering.

Youhei Kawamura (Member)

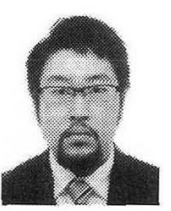

He received the B.E., M.E. and Ph.D. degrees in mineral resources development engineering from Hokkaido University, Sapporo, Japan in 1998, 2000 and 2003 respectively. Since 2003, he has been with Tsukuba University, where he is currently a research associate in Division of Intelligent Interaction Technologies. His research interests are geo-measurement engineering and mineral resources development engineering. 